\title{
CURVAS DE BIOMETRÍA FETAL CON EDAD GESTACIONAL DETERMINADA POR ECOGRAFÍA DE PRIMER TRIMESTRE. CLIINICA ALEMANA DE SANTIAGO
}

\author{
Julio Astudillo D. ${ }^{1}$, Masami Yamamoto C. ${ }^{1}$, Jorge Carrillo T. ${ }^{1}$, Max Polanco G. ${ }^{1}$, Alfre- \\ do Hernández A. ${ }^{1}$, Daniel Pedraza S. ${ }^{1}$, Paris Valentini Ch. ${ }^{1}$, Juan Valdivia M. ${ }^{1}$, Alvaro \\ Insunza F. ${ }^{1}$
}

1 Unidad de Medicina Materno Fetal, Departamento de Obstetricia y Ginecología, Clínica Alemana de Santiago, Universidad del Desarrollo.

\section{RESUMEN}

Antecedentes: La gran mayoría de las curvas ultrasonográficas de crecimiento intrauterino han sido basadas en una edad gestacional $(E G)$ determinada por fecha de última menstruación segura y confiable, la que es discordante con la ecografía de primer trimestre hasta en un 20\%. Objetivo: Presentación de un normograma para diámetro biparietal (DBP), circunferencia abdominal $(C A)$ y fémur $(F)$, en embarazos únicos con EG calculada por ecografía de primer trimestre. Métodos: En la Unidad de Ultrasonografía de la Clínica Alemana de Santiago, durante 2000 y 2004, se analizaron todos los embarazos simples con ecografía de primer trimestre, definida por embrión vivo, único, con longitud céfalo-nalgas entre 3 y $84 \mathrm{~mm}$. Se revisaron todos los informes ecográficos realizados durante el mismo embarazo, con informe de DBP, CA y F. Se seleccionaron 4.762 ecografías realizadas a 2.614 embarazadas. Se comparó los resultados con curvas de referencia nacional e internacional. Resultados: EI DBP de nuestra población a las 22 semanas es significativamente mayor que las publicadas por Chitty y Vaccaro, y similares a las de Kurmanavicius y Snijders; mientras que la CA es mayor y el fémur es similar a las demás curvas de referencia. Conclusión: Las curvas obtenidas y determinadas por la ecografía de primer trimestre son diferentes con las curvas de referencias comparadas. Esto deberá considerarse dado que la ecografía precoz para el cálculo de la edad gestacional se ha generalizado en la práctica obstétrica actual.

\section{PALABRAS CLAVE: Biometría fetal, curvas de referencia, crecimiento fetal, edad gestacional, ultrasonografía}

\section{SUMMARY}

Background: Several reference charts for fetal biometry are published in the literature. The choice may affect directly on diagnosis of IUGR. Most of these charts defined gestational age by last menstrual period (LMP), nevertheless, LMP has shown to be discordant with first trimester biometry in up to $20 \%$ of the cases in the best of clinical situations. Objective: To build a normogram of fetal biometry based on first trimester CRL and not on LMP. Methods: Between 2000 and 2004, 20400 scans were performed. First trimester CRL was used for dating second and third trimester biometries. 4762 scans in 2614 pregnant women were used to build our normal biometry charts. Our results were compared to other reference charts. Results: BDP at 22 weeks'GA form our population was significantly greater than that from Chitty and Vaccaro and similar to 
Kurmanavicius y Snijders. The AC of our population was greater and FL was similar to those of reference charts. Conclusion: The fetal biometry of our population was statistically different from that of local and international reference charts. This is important as gestational age is determined by first trimester ultrasound for obstetrical use.

\section{KEY WORDS: Fetal biometry, reference charts, fetal growth, gestational age, ultrasound}

\section{INTRODUCCIÓN}

Las curvas normales para la realización de la biometría fetal son de gran importancia para diagnosticar restricción de crecimiento, la condición de grande para la edad gestacional o ciertas malformaciones o enfermedades que presentan anomalías en el tamaño de los segmentos corporales. Por otra parte, se ha demostrado la importancia del uso de curvas adecuadas para cada medio local (1) Por este motivo, es importante disponer de curvas nacionales adecuadas que hayan sido construidas con la metodología apropiada.

Las curvas internacionales más conocidas son las de Chitty $(2,3,4)$, Kurmanavicius y Snijders $(5,6)$, las cuales son citadas en la mayoría de los libros de ultrasonografía y utilizadas como curvas de referencia en programas profesionales de informes ecográficos. Localmente destacan las curvas de Vaccaro, realizadas en 1991 (7). El problema de las curvas de referencia, en la cual debe expresarse la normalidad de las medidas a lo largo de la gestación, es justamente la datación. De hecho, la información que demostró que la edad gestacional es más precisa por ecografía de primer trimestre que por una fecha de última regla (FUR) segura y confiable se publicó después de la construcción de estas curvas (8). Los autores de estas curvas han utilizado como criterio de inclusión una FUR segura y confiable, y han excluído aquellos casos que a las 18 semanas no tenían biometría acorde. La práctica obstétrica actual se basa en la corroboración de la edad gestacional (EG) por la ecografía de primer trimestre, lo que hace que no sea el mismo sistema de determinación de la EG que las curvas con las cuales los fetos de nuestra población son comparados. Finalmente, la resolución de los equipos de ultrasonografía de la última década son completamente diferentes a los utilizados en los años 80 , por lo que es valedero reevaluar las curvas de normalidad, especialmente aquellas que miden partes blandas como la circunsferencia abdominal.

El objetivo de esta investigación es construir curvas de biometría fetal con equipos modernos y determinación de la EG con ecografías de primer trimestre.

\section{MATERIAL Y MÉTODO}

La Unidad de Medicina Materno Fetal de la Clínica Alemana de Santiago cuenta con sistemas de autoevaluación periódica. Durante los años 2000 y 2004 se realizaron 20.400 ecografías obstétricas, utilizando equipos de ultrasonografía Acuson Sequoia y General Electric Voluson 730. Los exámenes fueron efectuados por 9 operadores con experiencia en la realización de ecografías obstétricas y con técnica estandarizada de medición. Los datos fueron almacenados en programa de base de datos File Maker Proß. Para la construcción de las tablas se seleccionaron los embarazos con feto único en los cuales se dispuso de una ecografía de primer trimestre efectuada en la institución. Estas fueron definidas como aquellas en que se encontró un embrión vivo, único, con una longitud céfalo-nalgas entre $3 \mathrm{~mm}$ y $84 \mathrm{~mm}$.

Posteriormente, se identificaron las pacientes de este grupo que tuvieran en el mismo embarazo al menos una ecografía con medición del diámetro biparietal (DBP), circunferencia abdominal (CA) y fémur $(F)$. Solo se consideraron las pacientes con 2 o menos ecografías con medición de dichos parámetros para evitar el sesgo de participación de cada paciente. Para efectos del estudio, se determinó la edad gestacional de las ecografías de segundo y tercer trimestre según la longitud cefalo-nalgas (LCN) encontrado en la ultrasonografía de primer trimestre de la curva de Hansmann (9) y no por la fecha de última menstruación, aunque ésta fuera confiable y segura. De esta forma, la EG al momento de las subsiguientes ecografías fueron recalculadas retrospectivamente por LCN de la ecografía de primer trimestre.

EI DBP se midió desde el borde proximal de cada hueso temporal, es decir, usando el criterio "externo-interno". Aunque es un criterio que se ha abandonado por muchos centros por la calidad de los nuevos equipos que ya no presentan sombra posterior de estructuras óseas, se decidió en nuestro centro continuar con esta forma de obtención de la medición para mantener el mismo estándar. La CA se obtuvo por dos mediciones ortogonales de los diámetros anteroposterior y transverso en un 
corte transversal del abdomen y utilizando la fórmula de $\pi \times(\mathrm{D} 1+\mathrm{D} 2) / 2$. Para la elección del corte adecuado se utilizaron los criterios habituales que incluyen la porción intraabdominal de la vena umbilical y el estómago evitando la visualización de los riñones y el corazón. Las mediciones se realizaron desde los bordes de la piel. El fémur se midió con una inclinación menor a 45으 para eliminar la distorsión del ángulo en la medición.

Se excluyeron los casos en que se observaron malformaciones que pudieran alterar las mediciones en la ecografía actual. En el caso de DBP se eliminaron los casos con encefaloceles o ventriculomegalias. En la medición de la CA, no se consideraron los casos con onfalocele, gastrosquisis y ascitis. En la medición del fémur se excluyeron los casos con evidente patología osteomuscular. No se excluyeron los casos que posteriormente presentaron restricción del crecimiento intrauterino (RCIU) o macrosomía fetal, pues sesgaría la curva hacia mediciones con menor dispersión.

Nuestras curvas fueron sometidas a test de normalidad a las 22 semanas, para poder compararlas con otras publicadas en la literatura. Cuando las curvas de referencia no publicaban la información necesaria para poder compararlas, se evaluó el porcentaje de nuestras mediciones que se obtenían bajo el límite inferior.

No se corroboró el peso al parto ni la edad gestacional o la patología obstétrica que pudo haber desarrollado posteriormente en nuestra población. Esto se debe a que el servicio de ecografía realiza exámenes a médicos externos, y a su vez, muchas pacientes que tienen el parto en la Institución realizan sus ecografías en otros lugares. No se realizó análisis de variabilidad interobservador.

Se compararon nuestras mediciones con las tablas de referencia más conocidas y utilizadas de Chitty $(2,3,4)$, Kurmanavicius $(5,6)$, Snijders (10) y Vaccaro (7). Para las tablas de Chitty y Kurmanavicius que publican el número de medidas, el promedio y la desviación estándar (DE), se utilizó el test de Student. Para las tablas de Snijders y Vaccaro, que no publican el número de medidas, por lo que no permiten aplicar test estadísticos para comparar las poblaciones, se calculó el porcentaje de nuestra población que cae bajo el percentil 5 .

\section{RESULTADOS}

Se presentan las curvas obtenidas de DBP, CA y $F$ realizadas sobre la base de la edad gestacional calculada por ecografía de primer trimestre. Se seleccionaron 4.762 ecografías realizadas a 2.614 pacientes. De ellas, 1.038, 1.004 y 572 pacientes tenían una, dos y tres ecografías respectivamente. Para la confección de las curvas se utilizaron 4.762 ecografías de 2614 embarazadas. Hubo un promedio de 176 mediciones por cada edad gestacional, con un rango de 24 a 339 . Solamente a las 14,15 , 39 y 40 semanas hubo menos de 100 mediciones. Se calcula mediana, p5 y p95 para cada EG del parámetro medido (DBP, CA y F).

En las Figuras 1, 2 y 3 se muestran los gráficos para los p5, p50 y p95 para DBP, CA y F. Las Tablas I, II y III muestran la información resumida de las mediciones obtenidas. La medida del percentil 5 y 95 tienden a ser artificiosos en la mayoría de los artículos, ya que muchos de ellos tienen curvas con menos de 100 mediciones (n) por EG. En estas publicaciones se ha utilizado el método restar o sumar la desviación estándar (DE) multiplicada por 1,645 para estimar el valor del p5 y p95 respectivamente, lo cual sólo es aplicable si la población tiene una distribución normal. En nuestra población, tanto la aplicación de este método como el valor real del percentil 5 dieron exactamente lo mismo en todas las edades gestacionales evaluadas $(22,28$ y 32 semanas), lo que concuerda con la distribución normal de las medidas. Las curvas presentadas en los gráficos fueron suavizadas por una regresión polinomial de tercer grado, en la que el coeficiente de correlación fue siempre mayor a 0,95 para todas las medidas (p5, p50 y p95). Se estudió la normalidad de nuestras mediciones con el Test de Kolmorogov-Smirnov a las 22 y 32 semanas. La distribución de las medidas de DBP $(p=0,002), C A(p=0,006)$ y $F(p<0,001)$ fueron normales. Se obtiene el promedio y la desviación estándar (DE) para cada EG y parámetro estudiado (DPB, CA y F).

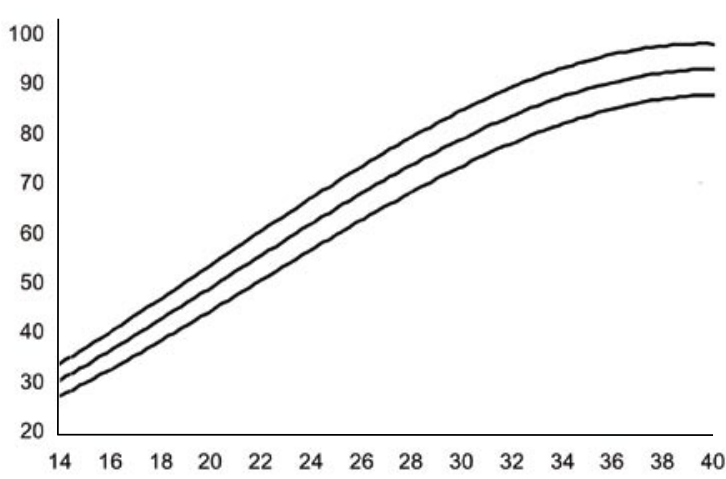

Figura 1. Diámetro biparietal $(\mathrm{mm})$ para la edad gestacional (semanas) determinada por ecografía de primer trimestre. Las líneas son suavizadas y representan los percentiles 5,50 y 95 para la edad gestacional. 


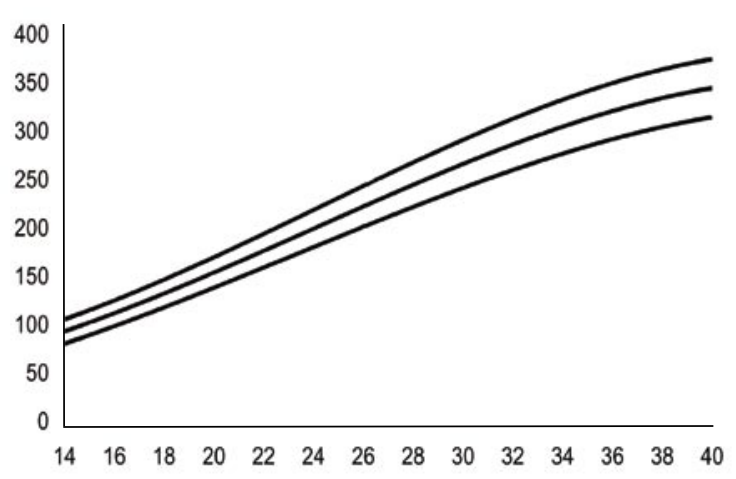

Figura 2. Circunferencia abdominal $(\mathrm{mm})$ para la edad gestacional (semanas) determinada por ecografía de primer trimestre. Las líneas son suavizadas y representan los percentiles 5,50 y 95 para la edad gestacional.

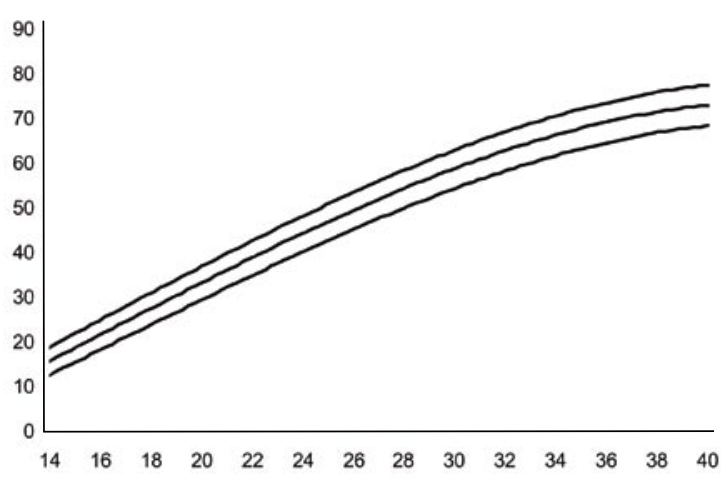

Figura 3. Fémur $(\mathrm{mm})$ para la edad gestacional (semanas) determinada por ecografía de primer trimestre. Las líneas son suavizadas y representan los percentiles 5,50 y 95 para la edad gestacional.

Tabla I

RESUMEN DE LAS MEDIDAS DE DIÁMETRO BIPARIETAL $(\mathrm{mm})$ A DISTINTAS EDADES GESTACIONALES (semanas)

\begin{tabular}{lllllll}
\hline $\mathrm{EG}$ & $\mathrm{n}$ & $\mathrm{X}$ & $\mathrm{DE}$ & $\mathrm{p5}$ & $\mathrm{p} 50$ & $\mathrm{p} 95$ \\
\hline 14 & 71 & 30,1 & 2,2 & 27 & 30 & 34 \\
15 & 81 & 33,3 & 2,5 & 29 & 33 & 37 \\
16 & 100 & 36,9 & 2,3 & 33 & 37 & 41 \\
17 & 128 & 40,5 & 2,4 & 36 & 40 & 44 \\
18 & 176 & 43,6 & 2,5 & 39 & 44 & 48 \\
19 & 227 & 46,4 & 2,5 & 42 & 46 & 51 \\
20 & 339 & 49,6 & 2,6 & 45 & 50 & 54 \\
21 & 317 & 52,6 & 2,8 & 48 & 53 & 57 \\
22 & 338 & 55,1 & 3 & 50 & 55 & 60 \\
23 & 240 & 58,1 & 3 & 53 & 58 & 63 \\
24 & 150 & 61,2 & 3,7 & 55 & 61 & 67 \\
25 & 114 & 64,4 & 3,3 & 59 & 64 & 70 \\
26 & 103 & 69,1 & 3,6 & 63 & 69 & 75 \\
27 & 125 & 72 & 3,1 & 67 & 72 & 77 \\
28 & 149 & 74,4 & 3,5 & 69 & 74 & 80 \\
29 & 186 & 77,8 & 3,1 & 73 & 78 & 83 \\
30 & 191 & 79,6 & 3,6 & 74 & 80 & 86 \\
31 & 220 & 81,7 & 3,5 & 76 & 82 & 88 \\
32 & 240 & 83,5 & 3 & 79 & 83 & 88 \\
33 & 249 & 85,9 & 3,4 & 80 & 86 & 92 \\
34 & 221 & 87,6 & 3,2 & 82 & 88 & 93 \\
35 & 231 & 89,2 & 3,5 & 83 & 89 & 95 \\
36 & 199 & 90,9 & 3,2 & 86 & 91 & 96 \\
37 & 184 & 92,5 & 3 & 88 & 92 & 97 \\
38 & 102 & 93,4 & 3,2 & 88 & 93 & 99 \\
39 & 57 & 93,6 & 3,4 & 88 & 94 & 99 \\
40 & 24 & 92 & 3,5 & 86 & 92 & 98 \\
\hline
\end{tabular}

EG: edad gestacional. X: promedio. DE : desvío estándar. p: percentil. 
Tabla II

\section{RESUMEN DE LAS MEDIDAS DE CIRCUNFERENCIA ABDOMINAL (mm) A DISTINTAS EDADES} GESTACIONALES (semanas)

\begin{tabular}{lllllll}
\hline EG & $\mathrm{n}$ & $\mathrm{X}$ & $\mathrm{DE}$ & $\mathrm{p} 5$ & $\mathrm{p} 50$ & $\mathrm{p} 95$ \\
\hline 14 & 71 & 89,7 & 7,4 & 77 & 90 & 102 \\
15 & 81 & 100,9 & 6,8 & 90 & 101 & 112 \\
16 & 100 & 112,3 & 8,3 & 99 & 112 & 126 \\
17 & 128 & 123,9 & 9,2 & 109 & 124 & 139 \\
18 & 176 & 135,6 & 9,4 & 120 & 136 & 151 \\
19 & 227 & 145,4 & 9,2 & 130 & 145 & 160 \\
20 & 339 & 156,3 & 9,7 & 140 & 156 & 172 \\
21 & 317 & 168,1 & 10,7 & 151 & 168 & 186 \\
22 & 338 & 176,6 & 10,3 & 160 & 177 & 194 \\
23 & 240 & 186,4 & 11 & 168 & 186 & 205 \\
24 & 150 & 197,2 & 12 & 177 & 197 & 217 \\
25 & 114 & 207,5 & 11,9 & 188 & 208 & 227 \\
26 & 103 & 224,5 & 13 & 203 & 224 & 246 \\
27 & 125 & 232,4 & 10,9 & 215 & 232 & 250 \\
28 & 149 & 243,8 & 14,7 & 220 & 244 & 268 \\
29 & 186 & 254,8 & 13,1 & 233 & 255 & 276 \\
30 & 191 & 264,9 & 16 & 239 & 265 & 291 \\
31 & 220 & 274,3 & 16,9 & 246 & 274 & 302 \\
32 & 240 & 284,3 & 16,8 & 257 & 284 & 312 \\
33 & 249 & 296,2 & 16,8 & 269 & 296 & 324 \\
34 & 221 & 306,5 & 16,4 & 280 & 307 & 334 \\
35 & 231 & 313,6 & 15,5 & 288 & 314 & 339 \\
36 & 199 & 324,2 & 18,5 & 294 & 324 & 355 \\
37 & 184 & 332,5 & 17,7 & 303 & 333 & 362 \\
38 & 102 & 338,9 & 19,8 & 306 & 339 & 371 \\
39 & 57 & 340,1 & 19 & 309 & 340 & 371 \\
40 & 24 & 337 & 16,7 & 310 & 337 & 364 \\
\hline & & & & & &
\end{tabular}

EG: edad gestacional. X: promedio. DE: desvío estándar. p: percentil.

\section{DISCUSIÓN}

Nuestras medidas de DBP son significativamente mayores a las 22 semanas que aquellas publicadas por Chitty (Student, $p<0,001$ ), similares a las publicadas por Kurmanavicius (Student, $p=0,32$ ) y similares pero no evaluables estadísticamente con la de Snijders por tener 40 mediciones y ausencia de una prueba que muestre una distribución normal de sus valores. Si se asume esto, se obtiene que no son diferentes (Student, $p=0,10$ ). Las tablas de referencia de Vaccaro no publican el número de mediciones por edad gestacional ni la DE, por lo que no puede compararse con nuestras curvas por este método. Sin embargo, su p5, p50 y p95 seleccionaron al $2,6 \%, 31 \%$ y $69 \%$ de nuestra po- blación, lo que muestra que nuestras mediciones son mayores.

A su vez, la medida de CA a las 22 semanas fue estadísticamente diferente, con una media 7 $\mathrm{mm}$ mayor que la curva de Kurmanavicius (Student $\mathrm{p}<2,5 \times 10^{-9}$ ). El resto de las curvas no pueden ser evaluadas por Student o un test no paramétrico, sin embargo, el p5 de Chitty, Snijders y Vaccaro seleccionan el $0,29 \%, 2,97 \%$ y $2,97 \%$ de nuestra población respectivamente. A su vez, nuestras mediciones bajo el p50 de las mismas curvas de referencia corresponden al $44,6 \%, 28,9 \%$ y $44,6 \%$ de la población. Por último, las mediciones bajo el p95 de las respectivas curvas corresponden al $97,3 \%$, $94,3 \%$ y $88,4 \%$ de nuestras mediciones. Esta comparación muestra que las $\mathrm{CA}$ a las 22 semanas son 
Tabla III

RESUMEN DE LAS MEDIDAS DE LONGITUD DE FÉMUR (mm) A DISTINTAS EDADES GESTACIONALES (semanas)

\begin{tabular}{|c|c|c|c|c|c|c|}
\hline EG & $\mathrm{n}$ & $x$ & $\mathrm{DE}$ & p5 & p50 & p95 \\
\hline 14 & 71 & 15,5 & 1,7 & 13 & 16 & 18 \\
\hline 15 & 81 & 18,5 & 2,1 & 15 & 18 & 22 \\
\hline 16 & 100 & 21,7 & 2,4 & 18 & 22 & 26 \\
\hline 17 & 128 & 25,0 & 2,2 & 21 & 25 & 29 \\
\hline 18 & 176 & 28,1 & 2,2 & 25 & 28 & 32 \\
\hline 19 & 227 & 30,9 & 2,1 & 28 & 31 & 34 \\
\hline 20 & 339 & 33,9 & 2,1 & 30 & 34 & 37 \\
\hline 21 & 317 & 36,6 & 2,2 & 33 & 37 & 40 \\
\hline 22 & 338 & 38,8 & 2,2 & 35 & 39 & 42 \\
\hline 23 & 240 & 41,1 & 2,2 & 37 & 41 & 45 \\
\hline 24 & 150 & 43,8 & 2,7 & 39 & 44 & 48 \\
\hline 25 & 114 & 46,8 & 2,3 & 43 & 47 & 51 \\
\hline 26 & 103 & 50,0 & 2,5 & 46 & 50 & 54 \\
\hline 27 & 125 & 51,9 & 2,5 & 48 & 52 & 56 \\
\hline 28 & 149 & 54,2 & 2,8 & 50 & 54 & 59 \\
\hline 29 & 186 & 56,5 & 2,6 & 52 & 57 & 61 \\
\hline 30 & 191 & 58,5 & 2,6 & 54 & 59 & 63 \\
\hline 31 & 220 & 60,9 & 2,7 & 56 & 61 & 65 \\
\hline 32 & 240 & 62,4 & 2,7 & 58 & 62 & 67 \\
\hline 33 & 249 & 64,5 & 2,7 & 60 & 65 & 69 \\
\hline 34 & 221 & 66,5 & 2,5 & 62 & 67 & 71 \\
\hline 35 & 231 & 67,8 & 2,6 & 64 & 68 & 72 \\
\hline 36 & 199 & 69,7 & 2,7 & 65 & 70 & 74 \\
\hline 37 & 184 & 71,3 & 2,5 & 67 & 71 & 75 \\
\hline 38 & 102 & 72,4 & 2,8 & 68 & 72 & 77 \\
\hline 39 & 57 & 72,6 & 2,8 & 68 & 73 & 77 \\
\hline 40 & 24 & 72,0 & 3,1 & 67 & 72 & 77 \\
\hline
\end{tabular}

EG: edad gestacional. X: promedio. DE: desvío estándar. p: percentil.

mayores en nuestra población que a la de las mencionadas tablas de referencia. Por último, nuestras mediciones del fémur a las 22 semanas no son distintas que las de Chitty $(p=0,02)$ y la de Kurmanavicius $(p=0,0017)$.

Las condiciones sociales y raciales locales son fundamentales para la confección de curvas normales para el uso de informes ecográficos. Así como en pediatría, las curvas de peso fetal deben adaptarse a las condiciones locales (11). En este sentido, la introducción de curvas nacionales para los pesos al nacimiento fue un gran avance (12). Pretendiendo seguir el mismo ejemplo, se publica la información de una gran cohorte de medidas fetales, con el fin de compararlas con otras de la literatura internacional y ponerlas a disposición para el medio nacional.

Otro gran aporte de esta publicación es que se han presentado las medidas en relación a la edad gestacional calculada por una ecografía de primer trimestre. Mongelli (8) ya ha publicado lo importante del uso de la ecografía para la determinación de la EG, pues hasta un $18 \%$ de las mujeres con FUR segura y confiable tienen discordancia con una ecografía. La gran mayoría de las publicaciones provienen de los años 80 , los cuales adolecen de 
una edad gestacional determinada por ecografía. Estos estudios han publicado sus resultados en base a fecha de última menstruación segura y confiable y todos han eliminado las mediciones cuando sus resultados no concuerdan con la edad gestacional. El estudio de Snijders y Nicolaides en 1994 (10) excluyó aquellos casos con peso al nacer $<\mathrm{p} 3$ y >p97, en los métodos. Las tablas de Kurmanavicius son más recientes y utilizan la confirmación por una ecografía precoz, pero se utilizan igualmente la FUR. Esto introduce un sesgo, que reduce falsamente la dispersión de las medidas. Posteriormente, en los años noventa, surgieron los estudios que publicaron las mediciones en embarazos obtenidos por técnicas de fertilización asistida (13). Ellos tienen la ventaja de conocer la edad gestacional precisa, pero carecen de un número de mediciones suficientes que permitan validar sus resultados. En nuestro estudio hay un número homogéneo de mediciones a lo largo todas las edades gestacionales y las curvas son más representativas de la población en general. En particular, esto se ve más afectado para el cálculo de los p5 y p95. En estos extremos de las curvas de normalidad, suelen haber pocos casos, y los resultados son dependientes de los pocos casos disponibles.

Una debilidad de nuestro estudio consiste en que no se conocen los resultados al parto de los recién nacidos, sin embargo, es importante declarar que no se habría excluído ningún recién nacido por tener un peso demasiado pequeño o grande. Aquellos estudios que han excluido los recién nacidos pequeños para la edad gestacional, excluyen para sus curvas las mediciones obtenidas de estos niños (10). Esto conduce a que el p10 calculado de esta curva sea el p10 de los niños eutróficos al nacer. La introducción de este sesgo genera posteriormente, un exceso de medidas bajo p10. Por este motivo, creemos que el hecho de desconocer el peso al parto de estos recién nacidos no tiene mayor relevancia para la validez de esta información.

Otra debilidad de este estudio es que no se dispone del respaldo de las imágenes de las mediciones realizadas, es decir, auditoria de imágenes y mediciones. En los primeros estudios internacionales, se pudo corroborar la calidad de las mediciones al revisar las fotografías. Suponemos que no debiera existir demasiado error ya que en la unidad las mediciones son realizadas por profesionales médicos especializados con experiencia. La única forma de corroborar que no existe error en la medición es mediante la realización de estudios de variabilidad interobservador en las mediciones.

La comparación de nuestras curvas con otras publicadas en la literatura mostró resultados interesantes. Existen tres formas para comparar curvas. En principio, una curva es una distribución de valores a distintas edades gestacionales. Se puede concebir como una suma de distribuciones. La forma más simple es evaluando la proporción de las mediciones que están bajo un percentil o una medida de dispersión determinada de una tabla de referencia, y ver si se observa una distribución similar. Por ejemplo, es posible que la curva no sea adecuada para la población si el $25 \%$ de las mediciones de fémur en nuestra población caen por debajo del percentil 10 de la curva de referencia. Un segundo método para la comparación de curvas es el uso de pruebas estadísticas para comparar las mediciones en de una población con otra, a una determinada edad gestacional. Por ejemplo, aplicar un T-Student para comparar las medidas del fémur obtenidas en la población en estudio con otra de referencia. La dificultad para realizar esto es que la gran mayoría de los estudios no publican toda la información para poder aplicar estas pruebas. Una tercera forma de comparación de curvas consiste en la utilización del Z-score, la cual consiste en calcular el Z-score de cada medición y evaluar si es diferente de una distribución normal por la prueba de Kolmogorov-Smirnov. No se utilizó Z-score en este estudio por ser lo suficientemente extenso para ameritar otra publicación.

El presente estudio acumula la experiencia de la Clínica Alemana de Santiago en la realización de biometrías fetales. La gran innovación de estas curvas es que se utilizó la ecografía de primer trimestre para la determinación de la edad gestacional al momento de la medición y no la fecha de última menstruación, lo que aporta mayor precisión al diagnóstico de la edad del embarazo.

La comparación de las curvas mostró que el DBP de nuestra población es mayor que la publicada por Chitty y Vaccaro y similar a la de Kurmanavicius y Snijders. Nuestras medidas de CA son mayores que la de Kurmanavicius y Vaccaro, y similares a la de Snijders. Nuestras mediciones de CA tienen una menor dispersión que la de Chitty, dado que el p5 y p95 de esta tabla selecciona al $0,27 \%$ y $97 \%$ de nuestra población. Nuestra curva de fémur no tiene diferencias con las curvas de Chitty ni Kurmanavicius.

\section{CONCLUSIÓN}

Estimamos que las diferencias encontradas justifican el uso de estas tablas como referencia nacional de fetometría ultrasonográfica. 


\section{BIBLIOGRAFÍA}

1. Comité de Expertos de la OMS. El estado físico: Uso e interpretación de la antropometría. OMS, Serie de informes técnicos. 1995; 854.

2. Chitty L, Altman D, Henderson A, Campbell. Charts of fetal size: 2. Head measurements. Br J Obstet Gynaecol 1994;101:35-43.

3. Chitty L, Altman D, Henderson A, Campbell. Charts of fetal size: 3 . Abdominal measurements. $\mathrm{Br} \mathrm{J}$ Obstet Gynaecol 1994; 101: 125-31.

4. Chitty L, Altman D, Henderson A, Campbell. Charts of fetal size: 4. Femur length. Br J Obstet Gynaecol 1994; 101:132-5.

5. Kurmanavicius J, Wright E, Royston P, Zimmerman $\mathrm{R}$, Huch $\mathrm{R}$, Huch $\mathrm{A}$, et al. Fetal ultrasound biometry: 1 . Head reference values. $\mathrm{Br} \mathrm{J}$ Obstet Gynaecol 1999;106:126-35.

6. Kurmanavicius J, Wright E, Royston P, Zimmerman $\mathrm{R}$, Huch R, Huch A, et al. Fetal ultrasound biometry: 2. Abdomen and femur length reference values. $\mathrm{Br} \mathrm{J}$ Obstet Gynaecol 1999;106:136-43.

7. Vaccaro H. Crecimiento fetal. Rev Chil Obstet Ginecol 1991;56(5):353-8.

8. Mongelli M, Wilcox M, Gardosi J. Estimating the date of confinement versus ultrasonographic biometry versus certain menstrual dates. Am J Obstet Gynecol 1996;174(1):278-81.

9. Hansmann M, Schuhmacher H, Foebus J, Voigt U. UItrasonic biometry of the fetal crown-rump length between 7 and 20 weeks gestation. Geburtshilfe Frauenheilkd 1979;39(8):656-66.

10. Snijders R, Nicolaides K. Fetal biometry at $14-40$ weeks' gestation. Ultrasound Obstet Gynecol 1994;4:3448.

11. Merialdi M, Caulfield LE, Zavaleta N, Figueroa A, Costigans KA, Dominici, Dipietro A. Fetal growth in Peru: comparisons with internacional fetal size charts and implications for fetal growth assessment. Ultrasound Obstet Gynecol 2005;26:123-8.

12. González R, Gómez R, Castro R, Nien JH, Merino P, Etchegaragy $\mathrm{A}$, et al. Curva Nacional de distribución de peso al nacer según edad gestacional. Chile, 1993 a 2000. Rev Med Chile 2004;132:1115-65.

13. Sladkevicious $P$, Saltvedt $S$, Almstrom $H$, Kublickas $M$, Grunewald C, Valentin L. Ultrasound dating at 12-14 weeks of gestation. A prospective cross validation of established dating formulae in in-vitro fertilized pregnancies. Ultrasound Obstet Gynecol 2005;26:504-11. 